\title{
Addendum to 39(1) Special section: Medical imaging and insights into the mechanics of heart and circulatory physiology and dysfunction
}

\author{
Martin Caon ${ }^{1}$
}

(C) Australasian College of Physical Scientists and Engineers in Medicine 2017

The following three articles did not complete the peer review process in time to be included with the other articles in the December 2016 issue's special section: Medical Imaging and Insights into the Mechanics of Heart and Circulatory Physiology and Dysfunction.

\section{Guest Editors:}

Kelvin Wong ${ }^{1}$, Barry Doyle ${ }^{2}$

${ }^{1}$ School of Medicine, Western Sydney University, Sydney, Australia

${ }^{2}$ Vascular Engineering, School of Mechanical and Chemical Engineering, The University of Western Australia, Perth, Australia
The aim of this Special Section is to source and publish high quality articles that involve heart mechanics and circulatory physiology. They fall within the area of interest of the Journal and/or the boundaries of the Special Section as they have a cardiovascular application.

(All articles were subject to final review and acceptance by the APESM Editor in Chief.)

Martin Caon

martin.caon@flinders.edu.au

1 Adelaide, Australia 